\title{
Evaluation and Identification of most Promising Genotypes for Varietal Development in Amaranthus (Amaranthus paniculatus L.)
}

\author{
Shiv Prakash Shrivastav*, C. B. Yadav, Vartika Singh and Virendra Maurya \\ Department of Genetics and Plant Breeding, Narendra Deva University of Agriculture and \\ Technology, Narendra Nagar (Kumarganj), Faizabad-224 229 (U.P.), India \\ *Corresponding author
}

\section{A B S T R A C T}

\section{Keywords}

Amaranthus (Amaranthus paniculatus L.), genetic variability, correlation, path

\section{Article Info}

Accepted:

15 December 2019

Available Online:

20 January 2020
The experiment on twenty three genotypes including three check varieties (GA 2, BGA 2 and RMA 7) of amaranthus (Amaranthus paniculatus L.) was conducted to work out the genetic variability, heritability, genetic advance, correlation, path coefficient and genetic divergence effects of their various attributes on seed yield. The analysis of variance revealed that mean squares due to treatments were highly significant for all characters except seed volume weight $(\mathrm{g} / 10 \mathrm{ml})$. The magnitude of phenotypic coefficient of variation was higher than corresponding genotypic coefficient of variation for all the characters. The high estimates (>20\%) of phenotypic (PCV) and genotypic (GCV) coefficients of variations were found in case of harvest index, biological yield/plant and low estimates $(<10 \%)$ were observed for protein content and seed volume weight. Correlation analysis indicated that seed yield per plant had high and positive association with inflorescence length, plant height and biological yield per plant, while negative association were found for days to 50\% flowering. Path analysis identified biological yield per plant and harvest index as important components having high order of direct and positive effect. Higher indirect and positive contributions on seed yield per plant were made by biological yield per plant via days to maturity followed by harvest index via protein content, inflorescence length via plant height. The characters identified above as important direct and indirect yield components merit due consideration in formulating selection strategy in amaranthus for selecting high yielding genotypes. The estimates of heritability in broad sense (> 75\%) were high for plant height, biological yield per plant, harvest index, days to maturity and inflorescence length. The high estimates of genetic advance in percent of mean (>20\%) were obtained for harvest index, biological yield per plant, plant height, number of branches per plant and inflorescence length. The contribution of ten characters towards divergence showed the highest contribution by plant height followed by biological yield per plant. High heritability and genetic advance indicate that the additive nature of gene action and reliability of those characters for selection and emerged as ideal traits for improvement through selection. 


\section{Introduction}

Amaranthus (Amaranthus paniculatus L.) is the Rabi season self pollinated, diploid $(2 \mathrm{n}=$ 32) crop. It belongs to the family Amaranthaceae, and it comes under $\mathrm{C}_{4}$ plant. The grain of amaranth was an important staple food of Central and South Americans, Indians in the $15^{\text {th }}$ century, but declined in importance when the growing of the crop was prohibited for religious reasons by the conquering Spaniards. In 1950, a biogeographer, named Sauer J.D., presented the first thorough investigation of the history and classification of the grain amaranths. He studied the grain amaranths (A. caudatus, A. cruentus, $A$. hypochondriacus), and indigenously cultivated A. cruentus and A. hypochondriacus can only be found in Guatemala and Mexico. On the other hand, Amaranthus caudatus is restricted to the Andean South America. Sauer proposed that the grain amaranths evolved independently from three different wild species of amaranths in different parts of the world. He later proposed an alternative hypothesis that the grain amaranths may have a common ancestor. In his studies, other species of amaranths were also described. From 1950 to 1967, few people were interested in studying amaranths except Sauer. It was not until 23 years later in the grain of amaranth was found to have an exceptionally high lysine content, compared with the levels of this essential amino acid in other major crops: wheat, corn and rice. This discovery marked amaranth as a promising crop to feed a hungry world. This attracted researchers in food science, crop science, genetics and botany to investigate this long-forgotten crop. The protein component of amaranth is quite close to the level recommended for balance diet in human. The protein in leaf is up to $14.3 \mathrm{~g} / \mathrm{kg}$ with an average of $12.4 \mathrm{~g} / \mathrm{kg}$. Grain amaranth (Amaranthus hypochondriacus L.) is a traditional crop of himalayan region generally cultivated as a mixed crop as well as part of subsistence agriculture in the hilly areas with comparatively lower rainfall under neglected agriculture conditions. With advent of green revolution, the cultivation of this crop has seen a conspicuous decline mainly due to the lack of awareness of its complementary nutritive value, non-availability of suitable high yielding varieties and lack of improved production techniques. To reverse this declining trend of cultivation, quick varietal improvement is being used as one of the important criteria in increasing the yield potential of this crop. Apart from that, traditionally this crop was grown by broadcasting the seeds thereby resulting in very low yield. Adoption of scientific cultivation practices including proper plant densities and other inputs are essential in maximizing grain yield. In this context, there is an imperative need for the breeders to evaluate and identify the stable genotypes that could give standard performance when tested under different plant density levels. Population density is a major environmental factor influencing the genetic parameters among the characters. Study of the extent of such influence of different plant density levels in these genetic parameters is required to formulate appropriate breeding strategies. Exploitation of heterosis and success in getting desirable segregants in breeding progarmme also depends to a greater extent on the degree of genetic divergence between the parents chosen. Further the genotypes should also perform stably.

The major grain-producing species are Amaranthus caudatus L., Amaranthus cruentus L. and A. hypochondriacus L. Amaranth uses the $\mathrm{C} 4$ pathway and has a high efficiency of $\mathrm{CO} 2$ utilization, high photosynthesis rate at high temperature and drought tolerance (Williams and Brenner, 1995), which make it possible to be grown in areas not suitable for other crops (Breene, 1991 and Lehman 1996). The nutritional value 
of Amaranth has been extensively studied (Becker et al., 1981; Teutonico et al., 1985; Martirosyan, 2001, 2003). Grain amaranths have higher protein having significantly higher lysine content than other cereal grains (Bressani, 1989; Lehman, 1989). Amaranth leaves are an excellent source of protein at its blossoming phase for various samples (Kadoshnikov et al., 2005). Chlorophyll a and $b$ present in leaves of higher plants are the main pigments of photosynthesis in the chloroplasts and have important functions in the absorption and exploitation of light energy, thereby influence photosynthetic efficiency (Pan; Dong, 1995). Chlorophyll content is positively associated with photosynthetic rate which increases biomass production and grain yield (Araus et al., 1997; Thomas et al., 2005). Therefore, understanding the genetic mechanism of chlorophyll content would be very important for yield improvement.

The production of amaranthus is low due to unavailability of superior genotypes with wider adaptability and disease resistance. So, there is need to identify superior genotypes having disease resistance and wider adaptability with maximum production and protein content. Taking these problems under consideration the present investigation was carried out to identify most promising genotypes for varietal development.

\section{Materials and Methods}

The study was designed to work out the status of association of different seed yield traits on seed yield per plant among 23 amaranthus genotypes at field experiment under present investigation was conducted during Rabi 2014-15 at the Student's Instructional Farm, N. D. University of Agriculture and Technology, Narendra Nagar (Kumarganj), Faizabad (U.P.) India. The experimental materials of studies comprised of 23 amaranthus genotypes including three check varieties viz., GA 2, BGA 2 and RMA 7. The experiment was laid out in Randomized Block Design. The observation were recorded on ten different seed yield traits viz., days to $50 \%$ flowering, days to maturity, plant height $(\mathrm{cm})$, inflorescence length $(\mathrm{cm})$, number of branches / plant, biological yield per plant (g), harvest index $(\%)$, seed volume weight $(\mathrm{g} / 10 \mathrm{ml})$, protein content $(\%)$ and seed yield per plant (g). Variability for different characters was estimated as suggested by Burton and de Vane (1953). Heritability in broad sense $\left(h^{2}\right)$ was calculated using the formula suggested by Hanson et.al. (1956). Expected genetic advance $(\overline{\mathrm{Ga}})$ was estimated by the method suggested by Johnson et al., (1955). The genetic divergence among 23 genotypes of amaranthus was worked out using Mahalanobis's (1936) D D $^{2}$ statistics.

\section{Results and Discussion}

The analysis of variance for the Randomized Block Design, accommodating 23 amaranthus accessions and including three checks, was done for each of the ten characters. The Analysis of variance is presented in Table 1. The analysis of variance revealed that mean squares due to treatments were highly significant for all characters except seed volume weight $(\mathrm{g} / 10 \mathrm{ml})$ for which it was not significant.

The Mean performance of 23 genotypes including three checks and range for ten characters are presented in Table 2. The days to $50 \%$ flowering ranged from 73.00 (RMA 58) to 81.00 days (RMA 7) with a general mean of 77.15 days. The best five genotypes for this trait in order of merit were RMA 58, SKGPA 86, Ambika GA 12-1, SKGPA 91 and SKGPA 72. The days to maturity ranged from 163.00 (SKNA 1003) to 168.00 days (KBGA 3 ) with a mean of 166.00 days. The best five genotypes for this trait in order of merit were 
SKNA 1003, KBGA 5, SKGPA 72, RMA 59 and SKGPA 68. The mean performance of plant height was $70.92 \mathrm{~cm}$ and it ranged from 48.53 (SKNA 401) to $94.60 \mathrm{~cm}$ (RMA 57). The best three genotypes for this trait in order of merit were RMA 57, BGA 4-9 and RGA 72. The minimum inflorescence length was exhibited by SKGPA $68(31.33 \mathrm{~cm})$, whereas, RMA $57(59.33 \mathrm{~cm})$ possessed highest inflorescence length. The mean performance was $45.65(\mathrm{~cm})$. The best five genotypes for this trait in order of merit were RMA 57, RMA 58, RGA 72, BGA 4-9 and RGA 11. The minimum number of branches per plant was exhibited by GA 2 (3.07), whereas, SKGPA 91 (6.93) possessed highest number of branches per plant. The mean performance was 4.65 branches per plant. The best five genotypes for this trait in order of merit were SKGPA 91, SKGPA 86, RGA 72, SKGPA 68 and SKGPA 72. The lowest and highest performance for biological yield per plant was recorded for SKNA $1003 \quad(50.67 \mathrm{~g})$ and SKGPA $86(176.00 \mathrm{~g})$, respectively with a mean performance of $103.71 \mathrm{~g}$. The best five genotypes for this trait in order of merit were SKGPA 86, BGA 8-5, GA 2, BGA 2 and RMA 58. The lowest harvest index was observed in case of SKGPA 86 (5.93\%), while the highest value was recorded by the KBGA $5(15.99 \%)$. The general mean for harvest index was $10.02 \%$. The best five genotypes for this trait in order of merit were KBGA 5, SKNA 1003, RGA 10, RMA 59 and SKNA 403 . The lowest seed volume weight $(\mathrm{g} / 10 \mathrm{ml})$ was observed in case of SKNA $1003(5.80 \mathrm{~g})$, while the highest value was recorded in RGA $72(6.30 \mathrm{~g})$ with a mean of $6.09 \mathrm{~g}$. The best three genotypes for this trait in order of merit were RGA 72, SKNA 403 and SKGPA 86. The protein content varied from $10.27 \%$ (SKGPA 68) to $13.43 \%$ (RGA 11) and the mean performance was $12.05 \%$. The best three genotypes for this trait in order of merit were RGA 11, SKGPA 74 and SKGPA 91. The highest seed yield per plant was observed in RMA $58(11.53 \mathrm{~g})$, while, lowest seed yield per plant was produced by SKGPA 72 (7.27 g). The mean performance of seed yield per plant was $9.30 \mathrm{~g}$. The best three genotypes for this trait in order of merit were RMA 58, RGA 72, BGA 8-5, Ambika GA 12-1 and SKGPA 86.

The phenotypic and genotypic coefficients of variation for all the ten characters have been given in Table 3. In general, the magnitude of phenotypic coefficient of variation was higher than corresponding genotypic coefficient of variation for all the characters. The high estimates (>20\%) of phenotypic (PCV) and genotypic (GCV) coefficients of variation were recorded in case of harvest index $(\mathrm{PCV}=32.06 \%, \quad \mathrm{GCV}=29.03 \%), \quad$ biological yield/plant $(\mathrm{PCV}=30.76 \%, \mathrm{GCV}=27.81 \%)$ and number of branches per plant ( $\mathrm{PCV}=25.36 \%$, $\mathrm{GCV}=20.56 \%)$. Moderates estimates $(<20 \%$ $>10 \%$ ) of PCV and GCV were noted for inflorescence length (PCV $=19.67 \%$, $\mathrm{GCV}=17.17 \%$ ), plant height ( $\mathrm{PCV}=19.44 \%$, $\mathrm{GCV}=18.23 \%$ ) seed yield/plant $(\mathrm{PCV}=16.59 \%, \mathrm{GCV}=9.27 \%)$ whereas, the low estimates $(<10 \%)$ of phenotypic and genotypic coefficients of variations were observed for protein content $(\mathrm{PCV}=6.62 \%$, $\mathrm{GCV}=5.26 \%$ ), seed volume weight (PCV $=3.11 \%, \mathrm{GCV}=1.25 \%$ ), days to $50 \%$ flowering ( $\mathrm{PCV}=2.99 \%, \mathrm{GCV}=2.53 \%)$, and days to maturity ( $\mathrm{PCV}=1.01 \%, \mathrm{GCV}=0.90 \%)$.

Heritability in broad sense and genetic advance in per cent of mean for all the ten yield contributing traits were estimated and findings are given in Table 3. High estimates of broad sense heritability (> 75\%) were recorded for plant height $(88.00 \%)$, biological yield per plant $(82.00 \%)$, harvest index $(82.00 \%)$, days to maturity $(80.00 \%)$ and inflorescence length $(76 \%)$. The moderate estimates of heritability $(50-75 \%)$ were observed for days to $50 \%$ flowering $(71 \%)$, number of branches/plant $(66.00 \%)$, protein 
content $(63 \%)$ while the low estimates of broad sense heritability were shown by seed yield per plant $(31.00 \%)$, seed volume weight $(16.00 \%)$. The high estimates of genetic advance in per cent of mean (>20\%) were recorded for harvest index (54.15\%), biological yield per plant $(51.79 \%)$, plant height $(35.23 \%)$, number of branches per plant (34.33\%), inflorescence length (30.86). while the seeds yield per plant $(10.66 \%)$, protein content $(8.63 \%)$, days to $50 \%$ flowering (4.39\%) and days to maturity (1.68\%) showed low estimate of genetic advance in per cent of mean.

The estimates of phenotypic and genotypic correlation coefficients among different characters of amaranthus are presented in the Table 4. Seed yield per plant showed highly significant and positive correlation with inflorescence length (0.4171), plant height (0.4121) and biological yield per plant (0.3896) while positive and non significant correlation with days to maturity (0.3364); seed yield / plant showed negative non significant correlation with days to $50 \%$ flowering (-0.1969) and protein content (0.0369). The available literature had also identified the above character as important associate of grain yield in amaranthus (Kumar et al., 2014; Akaneme and Ani, 2013; Singh et al., 2012). Days to $50 \%$ flowering exhibited highly significant and negative correlation with only number of branches per plant (0.4109) and negative correlation with seed yield per plant (-0.1969), seed volume weight $(-0.0942)$. Days to maturity showed positive and highly significant correlation with inflorescence length (0.7093), plant height (0.7015), biological yield/plant (0.6443) and seed volume weight (0.3252) and negative and highly significant correlation with harvest index (-0.5262) while negative correlation with number of branches per plant $(-0.0834)$ and among the other characters showed non significant. Plant height $(\mathrm{cm})$ had highly significant and positive correlation with inflorescence length (0.8691), days to maturity $(0.7015)$ biological yield per plant (0.4440), seed yield per plant (41.21) and negative significant correlation with harvest index (-0.2991). The inflorescence length showed positive and highly significant correlation with plant height (0.8691), days to maturity (0.7093) biological yield per plant (0.4510), seed yield per plant (0.4171) and positive correlation with protein content (0.2231), seed volume weight (0.2059), days to $50 \%$ flowering $(0.0581)$ while negative highly significant correlation with harvest index (-0.3174) and negative and non significant correlation with number of branches per plant $(-0.0134)$. The number of branches per plant showed positive correlation with protein content $(0.1567)$, seed volume weight (0.1445), biological yield per plant (0.0408), plant height (0.0384), harvest index (0.0063) and negative significant correlation with days to maturity (-0.0834), inflorescence length (-0.0134) while negative highly significant correlation with days to $50 \%$ flowering (-0.4109). The biological yield per plant exhibited positive and highly significant correlation with days to maturity (0.6443), inflorescence length (0.4510), plant height (0.4440), seed yield per plant (0.3896) and positive correlation with seed volume weight (0.1502), number of branches/plant (0.0408) while highly significant and negative correlation with harvest index (-0.8099) and negative correlation with protein content ($0.2175)$, days to $50 \%$ flowering (-0.0234). Harvest index showed positive correlations with protein content $(0.1659)$, seed yield/plant (g) (0.0626) and negative correlation with seed volume weight (-0.0336), days to $50 \%$ flowering (-0.1090) while negative and highly significant correlation with biological yield/plant (-0.8099), days to maturity (0.5262), inflorescence length (-0.3174) and negative and significant correlation with only plant height $(-0.2991)$. 
Table.1 Analysis of variance for ten characters in Amaranthus

\begin{tabular}{|c|c|c|c|c|}
\hline \multirow[t]{3}{*}{ S. No. } & \multirow[t]{2}{*}{ Characters } & \multicolumn{3}{|c|}{ Source of variation } \\
\hline & & Replications & Treatments & Error \\
\hline & Degree of freedom & 2 & 22 & 44 \\
\hline 1 & Days to $50 \%$ flowering & 1.58 & $12.96 * *$ & 1.55 \\
\hline 2 & Days to maturity & 0.19 & $7.36 * *$ & 0.55 \\
\hline 3 & Plant height $(\mathrm{cm})$ & 29.91 & $524.51 * *$ & 22.87 \\
\hline 4 & Inflorescence length $(\mathrm{cm})$ & 0.26 & $203.47 * *$ & 19.24 \\
\hline 5 & Number of branches per plant & 0.77 & $3.22 * *$ & 0.48 \\
\hline 6 & Biological yield per plant (g) & 25.09 & $2680.68 * *$ & 185.78 \\
\hline 7 & Harvest index $(\%)$ & 2.87 & $27.22 * *$ & 1.86 \\
\hline 8 & Seed volume weight $(\mathrm{g} / 10 \mathrm{ml})$ & 0.07 & 0.05 & 0.03 \\
\hline 9 & Protein content $(\%)$ & 0.34 & $1.44 * *$ & 0.23 \\
\hline 10 & Seed yield per plant (g) & 1.70 & $3.87 * *$ & 1.64 \\
\hline
\end{tabular}

** Significant at $1 \%$ probability level. 
Table.2 Adjusted mean of genotypes and checks, range and least significant differences for ten characters in Amaranthus

\begin{tabular}{|c|c|c|c|c|c|c|c|c|c|c|c|}
\hline S. N. & Genotypes & $\begin{array}{c}\text { Days to 50\% } \\
\text { Flowering }\end{array}$ & Days to Maturity & $\begin{array}{l}\text { Plant Height } \\
(\mathbf{c m})\end{array}$ & $\begin{array}{l}\text { Inflorescence Length } \\
(\mathbf{c m})\end{array}$ & Branches/ Plant & $\begin{array}{c}\text { Biological Yield/ } \\
\text { Plant }\end{array}$ & $\begin{array}{c}\text { harvest } \\
\text { Index (\%) }\end{array}$ & $\begin{array}{c}\text { Seed Volume } \\
\text { Weight } \\
(\mathrm{g} / 10 \mathrm{ml})\end{array}$ & $\begin{array}{c}\text { Protein } \\
\text { Content } \\
(\%)\end{array}$ & $\begin{array}{l}\text { Seed Yield/ } \\
\text { Plant (g) }\end{array}$ \\
\hline 1 & SKGPA 68 & 75.6667 & 164.6667 & 49.8000 & 31.3333 & 5.6667 & 110.6666 & 8.8367 & 6.2000 & 10.2667 & 9.6333 \\
\hline 2 & SKGPA 86 & 74.6667 & 167.3333 & 74.9333 & 50.1333 & 6.6667 & 176.0000 & 5.9333 & 6.2333 & 12.1667 & 10.2667 \\
\hline 3 & SKGPA 74 & 77.6667 & 165.3333 & 69.0667 & 41.2667 & 5.4000 & 102.0000 & 8.0000 & 6.1333 & 13.2333 & 8.0333 \\
\hline 4 & SKGPA 91 & 75.3333 & 166.0000 & 76.6000 & 48.6000 & 6.9333 & 99.0000 & 8.5467 & 6.0000 & 13.2000 & 8.3667 \\
\hline 5 & SKGPA 72 & 75.3333 & 163.6667 & 60.4667 & 39.9333 & 5.8000 & 71.3333 & 10.3133 & 6.0333 & 11.3333 & 7.2667 \\
\hline 6 & SKNA 1003 & 75.3333 & 163.0000 & 54.5333 & 33.6000 & 5.0000 & 50.6667 & 15.2500 & 5.8000 & 12.4333 & 7.5667 \\
\hline 7 & RGA 10 & 75.6667 & 164.6667 & 59.4667 & 38.2667 & 4.2000 & 71.6667 & 14.5233 & 6.0333 & 12.3333 & 9.9333 \\
\hline 8 & RGA 72 & 76.0000 & 167.6667 & 90.2000 & 57.2000 & 6.0667 & 114.6666 & 12.2167 & 6.3000 & 12.0667 & 11.3000 \\
\hline 9 & RGA 11 & 79.0000 & 167.0000 & 81.2000 & 52.8667 & 5.4000 & 91.0000 & 11.2633 & 6.1000 & 13.4333 & 9.8333 \\
\hline 10 & RMA 57 & 79.0000 & 168.0000 & 94.6000 & 59.3333 & 4.1333 & 121.0000 & 7.8700 & 6.0667 & 12.2667 & 9.3667 \\
\hline 11 & RMA 58 & 73.3333 & 167.3333 & 80.7333 & 57.9333 & 4.1333 & 123.6667 & 9.2000 & 6.1333 & 12.4667 & 11.5333 \\
\hline 12 & RMA 59 & 76.6667 & 164.0000 & 66.4667 & 41.0000 & 4.1333 & 66.0000 & 14.4733 & 6.2000 & 12.1333 & 9.0667 \\
\hline 13 & KBGA 5 & 77.6667 & 163.3333 & 56.2667 & 37.3333 & 4.2667 & 58.3333 & 15.9900 & 6.0333 & 12.1000 & 9.3333 \\
\hline 14 & BGA 4-9 & 77.3333 & 167.6667 & 93.8667 & 55.0667 & 4.4667 & 121.0000 & 8.1400 & 6.1000 & 11.8667 & 9.7333 \\
\hline 15 & BGA 8-5 & 76.6667 & 167.0000 & 74.8667 & 50.1333 & 4.2667 & 136.6666 & 7.9567 & 6.1000 & 11.4333 & 10.7333 \\
\hline 16 & SKNA 403 & 79.6667 & 165.6667 & 62.6000 & 41.2667 & 4.3333 & 62.6667 & 14.4067 & 6.2667 & 12.4000 & 8.7333 \\
\hline 17 & SKNA 401 & 78.6667 & 165.6667 & 48.5333 & 32.8667 & 3.9333 & 103.3334 & 8.0967 & 5.8667 & 11.7000 & 7.6667 \\
\hline 18 & MGA 15 & 80.3333 & 166.3333 & 82.5333 & 51.6667 & 3.8000 & 120.0000 & 8.0233 & 5.9000 & 11.7667 & 9.1333 \\
\hline 19 & $\begin{array}{c}\text { Ambika GA } \\
12-1\end{array}$ & 74.6667 & 164.6667 & 70.6667 & 43.9333 & 4.3333 & 106.6667 & 10.1467 & 6.1333 & 11.9000 & 10.6000 \\
\hline 20 & KBGA 3 & 76.0000 & 168.0000 & 70.8000 & 49.2667 & 3.4667 & 107.6666 & 8.8467 & 6.1667 & 11.9333 & 9.0333 \\
\hline 21 & GA 2 (c) & 79.0000 & 167.6667 & 76.0000 & 50.5333 & 3.0667 & 133.6667 & 6.6833 & 6.2000 & 11.2000 & 8.9000 \\
\hline 22 & RMA 7 (c) & 81.0000 & 166.3333 & 78.1333 & 45.8000 & 3.2667 & 106.6666 & 9.1067 & 5.9667 & 11.5667 & 9.5000 \\
\hline \multirow[t]{10}{*}{23} & BGA 2 (c) & 79.6667 & 165.3333 & 58.8667 & 40.6667 & 4.2667 & 131.0000 & 6.5133 & 6.1000 & 11.9000 & 8.4333 \\
\hline & Mean & 77.1449 & 165.9275 & 70.9217 & 45.6522 & 4.6522 & 103.7101 & 10.0146 & 6.0899 & 12.0478 & 9.3029 \\
\hline & C.V. & 1.6135 & 0.4478 & 6.7434 & 9.6071 & 14.8528 & 13.1424 & 13.6105 & 2.8511 & 4.0086 & 13.7603 \\
\hline & F ratio & 8.3673 & 13.3365 & 22.9316 & 10.5776 & 6.7479 & 14.4296 & 14.6492 & 1.5725 & 6.1712 & 2.3604 \\
\hline & F prob. & 0.0000 & 0.0000 & 0.0000 & 0.0000 & 0.0000 & 0.0000 & 0.0000 & 0.0995 & 0.0000 & 0.0076 \\
\hline & S.E. & 0.7187 & 0.4290 & 2.7612 & 2.5322 & 0.3989 & 7.8693 & 0.7870 & 0.1002 & 0.2788 & 0.7391 \\
\hline & C.D. $5 \%$ & 2.0483 & 1.2226 & 7.8699 & 7.2172 & 1.1370 & 22.4289 & 2.2430 & 0.2857 & 0.7947 & 2.1065 \\
\hline & C.D. $1 \%$ & 2.7363 & 1.6333 & 10.5132 & 9.6412 & 1.5189 & 29.9621 & 2.9963 & 0.3817 & 1.0616 & 2.8140 \\
\hline & $\begin{array}{l}\text { Range } \\
\text { Lowest }\end{array}$ & 73.3333 & 163.0000 & 48.5333 & 31.3333 & 3.0667 & 50.6667 & 5.9333 & 5.8000 & 10.2667 & 7.2667 \\
\hline & $\begin{array}{l}\text { Range } \\
\text { Highest }\end{array}$ & 81.0000 & 168.0000 & 94.6000 & 59.3333 & 6.9333 & 176.0000 & 15.9900 & 6.3000 & 13.4333 & 11.5333 \\
\hline
\end{tabular}


Table.3 Estimate of coefficient of variation, $\mathrm{h}^{2}$ (broad sense) and genetic advance in per cent of mean in Amaranthus

\begin{tabular}{|c|c|c|c|c|c|c|c|}
\hline \multirow[t]{2}{*}{ S. No. } & \multirow[t]{2}{*}{ Characters } & \multirow[t]{2}{*}{ Range } & \multirow[t]{2}{*}{ Mean } & \multicolumn{2}{|c|}{ Coefficient of variation } & \multirow{2}{*}{$\begin{array}{c}\text { Heritability in } \\
\text { broad sense } \\
(\%)\end{array}$} & \multirow{2}{*}{$\begin{array}{c}\text { Genetic } \\
\text { advance in } \\
\text { per cent of } \\
\text { mean }\end{array}$} \\
\hline & & & & PCV & GCV & & \\
\hline $\mathbf{1}$ & Days to $50 \%$ flowering & $73.00-81.00$ & 77.00 & 2.99 & 2.53 & 71 & 4.39 \\
\hline 2 & Days to maturity & $163.00-168.00$ & 166.00 & 1.01 & 0.90 & 80 & 1.68 \\
\hline 3 & Plant height $(\mathrm{cm})$ & $48.53-94.60$ & 70.92 & 19.44 & 18.23 & 88 & 35.23 \\
\hline 4 & $\begin{array}{l}\text { Inflorescence } \\
\text { length }(\mathrm{cm})\end{array}$ & $31.33-59.33$ & 45.65 & 19.67 & 17.17 & 76 & 30.86 \\
\hline 5 & $\begin{array}{l}\text { Number of branches } \\
\text { per plant }\end{array}$ & $3.07-6.93$ & 4.65 & 25.36 & 20.56 & 66 & 34.33 \\
\hline 6 & $\begin{array}{l}\text { Biological yield per } \\
\text { plant }(\mathrm{g})\end{array}$ & $50.67-176.00$ & 103.71 & 30.76 & 27.81 & 82 & 51.79 \\
\hline 7 & Harvest index (\%) & $5.93-15.99$ & 10.02 & 32.06 & 29.03 & 82 & 54.15 \\
\hline 8 & $\begin{array}{l}\text { Seed volume weight } \\
(\mathrm{g} / 10 \mathrm{ml})\end{array}$ & $5.80-6.30$ & 6.09 & 3.11 & 1.25 & 16 & 1.03 \\
\hline 9 & Protein content $(\%)$ & $10.27-13.43$ & 12.05 & 6.62 & 5.26 & 63 & 8.63 \\
\hline 10 & Seed yield per plant (g) & $7.27-11.53$ & 9.30 & 16.59 & 9.27 & 31 & 10.66 \\
\hline
\end{tabular}


Table.4 Estimates of Phenotypic correlation between different characters in Amaranthus

\begin{tabular}{|c|c|c|c|c|c|c|c|c|c|c|c|}
\hline $\begin{array}{l}\text { S. } \\
\text { N. }\end{array}$ & Characters & $\begin{array}{c}\text { Days to } \\
50 \% \\
\text { Flowering }\end{array}$ & $\begin{array}{l}\text { Days to } \\
\text { Maturity }\end{array}$ & $\begin{array}{l}\text { Plant height } \\
\text { (cm) }\end{array}$ & $\begin{array}{l}\text { Inflorescence } \\
\text { Length }(\mathrm{cm})\end{array}$ & $\begin{array}{l}\begin{array}{c}\text { Number } \\
\text { of } \\
\text { branches } \\
\text { per plant }\end{array}\end{array}$ & $\begin{array}{l}\text { Biological } \\
\text { Yield/ } \\
\text { Plant (g) }\end{array}$ & $\begin{array}{c}\text { Harvest } \\
\text { index }(\%)\end{array}$ & $\begin{array}{c}\text { Seed Volume } \\
\text { Weight }(\mathrm{g} / 10 \mathrm{ml})\end{array}$ & $\begin{array}{c}\text { Protein } \\
\text { Content } \\
(\%)\end{array}$ & $\begin{array}{l}\text { Seed Yield/ } \\
\text { Plant (g) }\end{array}$ \\
\hline 1 & $\begin{array}{l}\text { Days to } 50 \% \\
\text { flowering }\end{array}$ & 1.0000 & 0.1354 & 0.0687 & 0.0581 & $-0.4109 * *$ & -0.0234 & -0.1090 & -0.0942 & 0.0001 & -0.1969 \\
\hline 2 & $\begin{array}{l}\text { Days to } \\
\text { maturity }\end{array}$ & & 1.0000 & $0.7015^{* *}$ & $0.7093 * *$ & -0.0834 & $0.6443 * *$ & $-0.5262 * *$ & $0.3252 * *$ & 0.0572 & $0.3364 * *$ \\
\hline 3 & $\begin{array}{l}\text { Plant height } \\
\quad(\mathrm{cm})\end{array}$ & & & 1.0000 & $0.8691 * *$ & 0.0384 & $0.4440 * *$ & $-0.2991 *$ & 0.1898 & 0.2200 & $0.4121 * *$ \\
\hline 4 & $\begin{array}{l}\text { Inflorescence } \\
\text { length }(\mathrm{cm})\end{array}$ & & & & 1.0000 & -0.0134 & $0.4510 * *$ & $-0.3174 * *$ & 0.2059 & 0.2231 & $0.4171 * *$ \\
\hline 5 & $\begin{array}{c}\text { Number of } \\
\text { branches per } \\
\text { plant }\end{array}$ & & & & & 1.0000 & 0.0408 & 0.0063 & 0.1445 & 0.1567 & 0.0216 \\
\hline 6 & $\begin{array}{l}\text { Biological } \\
\text { yield per } \\
\text { plant (g) }\end{array}$ & & & & & & 1.0000 & $-0.8099 * *$ & 0.1502 & -0.2175 & $0.3896 * *$ \\
\hline 7 & $\begin{array}{c}\text { Harvest } \\
\text { index }(\%)\end{array}$ & & & & & & & 1.0000 & -0.0336 & 0.1659 & 0.0626 \\
\hline 8 & $\begin{array}{c}\text { Seed volume } \\
\text { weight (g/10 } \\
\mathrm{ml})\end{array}$ & & & & & & & & 1.0000 & 0.0173 & 0.1656 \\
\hline 9 & $\begin{array}{c}\text { Protein } \\
\text { content }(\%)\end{array}$ & & & & & & & & & 1.0000 & -0.0369 \\
\hline 10 & $\begin{array}{c}\text { Seed yield } \\
\text { per plant }(\mathrm{g})\end{array}$ & & & & & & & & & & 1.0000 \\
\hline
\end{tabular}

$*, * *$ indicate significant at $5 \%$ and $1 \%$ probability level, respectively. 
Table.5 Phenotypic direct and indirect effect of different characters on seed yield per plant in Amaranthus

\begin{tabular}{|c|c|c|c|c|c|c|c|c|c|c|c|}
\hline S.N. & Characters & $\begin{array}{l}\text { Days to } \\
50 \% \\
\text { Flowering }\end{array}$ & $\begin{array}{l}\text { Days to } \\
\text { Maturity }\end{array}$ & $\begin{array}{l}\text { Plant } \\
\text { height } \\
\text { (cm) }\end{array}$ & $\begin{array}{l}\text { Inflorescence } \\
\text { Length }(\mathrm{cm})\end{array}$ & $\begin{array}{c}\text { Number } \\
\text { of } \\
\text { branches } \\
\text { per } \\
\text { plant }\end{array}$ & $\begin{array}{l}\text { Biological } \\
\text { Yield/ } \\
\text { Plant (g) }\end{array}$ & $\begin{array}{c}\text { Harvest } \\
\text { index } \\
(\%)\end{array}$ & $\begin{array}{c}\text { Seed Volume } \\
\text { Weight }(g / 10 m l \\
)\end{array}$ & $\begin{array}{c}\text { Protein } \\
\text { Content } \\
(\%)\end{array}$ & $\begin{array}{c}\text { Correlation } \\
\text { with Seed } \\
\text { Yield/ } \\
\text { Plant (g) }\end{array}$ \\
\hline 1 & $\begin{array}{c}\text { Days to } 50 \% \\
\text { flowering }\end{array}$ & -0.1037 & -0.0140 & $\begin{array}{c}- \\
0.0071\end{array}$ & -0.0060 & 0.0426 & 0.0024 & 0.0113 & 0.0098 & 0.0000 & -0.1969 \\
\hline 2 & $\begin{array}{l}\text { Days to } \\
\text { maturity }\end{array}$ & -0.0035 & -0.0258 & $\begin{array}{c}- \\
0.0181\end{array}$ & -0.0183 & 0.0022 & -0.0167 & 0.0136 & -0.0084 & -0.0015 & 0.3364 \\
\hline 3 & $\begin{array}{l}\text { Plant height } \\
\text { (cm) }\end{array}$ & 0.0067 & 0.0688 & 0.0980 & 0.0852 & 0.0038 & 0.0435 & -0.0293 & 0.0186 & 0.0216 & 0.4121 \\
\hline 4 & $\begin{array}{l}\text { Inflorescence } \\
\text { length }(\mathrm{cm})\end{array}$ & 0.0109 & 0.1333 & 0.1634 & 0.1880 & -0.0025 & 0.0848 & -0.0597 & 0.0387 & 0.0419 & 0.4171 \\
\hline 5 & $\begin{array}{l}\text { Number of } \\
\text { branches } \\
\text { per plant }\end{array}$ & 0.0293 & 0.0060 & $\begin{array}{c}- \\
0.0027\end{array}$ & 0.0010 & -0.0714 & -0.0029 & -0.0004 & -0.0103 & -0.0112 & 0.0216 \\
\hline 6 & $\begin{array}{l}\text { Biological } \\
\text { yield per } \\
\text { plant }(\mathrm{g})\end{array}$ & -0.0260 & 0.7148 & 0.4926 & 0.5003 & 0.0452 & 1.1094 & -0.8984 & 0.1666 & -0.2413 & 0.3896 \\
\hline 7 & $\begin{array}{c}\text { Harvest } \\
\text { index }(\%)\end{array}$ & -0.1121 & -0.5409 & $\begin{array}{c}- \\
0.3074\end{array}$ & -0.3263 & 0.0064 & -0.8324 & 1.0279 & -0.0345 & 0.1706 & 0.0626 \\
\hline 8 & $\begin{array}{l}\text { Seed volume } \\
\text { weight (g/10 } \\
\text { ml) }\end{array}$ & 0.0014 & -0.0047 & $\begin{array}{c}- \\
0.0028\end{array}$ & -0.0030 & -0.0021 & -0.0022 & 0.0005 & -0.0145 & -0.0003 & 0.1656 \\
\hline 9 & $\begin{array}{c}\text { Protein } \\
\text { content }(\%)\end{array}$ & 0.0000 & -0.0010 & $\begin{array}{c}- \\
0.0037\end{array}$ & -0.0037 & -0.0026 & 0.0036 & -0.0028 & -0.0003 & -0.0168 & -0.0369 \\
\hline
\end{tabular}

R SQUARE $=0.6238$, RESIDUAL EFFECT $=0.6134$, Bold figures indicate direct effect 
Table.6 Clustering pattern of 23 amaranthus genotyps \& on the basis of Mahalanobis's $D^{2}$ statistic

\begin{tabular}{|c|c|c|}
\hline Cluster No. & No. of Genotypes & Genotypes \\
\hline Cluster 1 & 2 & SKGPA 74, SKGPA 91 \\
\hline Cluster $\mathbf{2}$ & 4 & SKGPA 86, RGA 12, RGA 11, RMA 58 \\
\hline Cluster 3 & 5 & SKGPA 72, SKNA 1003, RGA 10, RMA 59, KBGA 5 \\
\hline Cluster $\mathbf{4}$ & 5 & SKGPA 68, SKNA 403, SKNA 401, Ambika GA 12-1, BGA 2(C) \\
\hline Cluster 5 & 7 & RMA 57, BGA 4-9, BGA 8-5, MGA 15, KBGA 3, GA 2(C), RMA 7 (C) \\
\hline
\end{tabular}

Table.7 Estimates of average intra and inter-cluster distances for the five clusters in Amaranthus

\begin{tabular}{|l|c|c|c|c|c|}
\hline Clusters & Cluster 1 & Cluster 2 & Cluster 3 & Cluster 4 & Cluster 5 \\
\hline Cluster 1 & $\mathbf{1 1 . 5 8}$ & 27.04 & 21.94 & 39.44 & 27.69 \\
\hline Cluster 2 & & $\mathbf{1 2 . 2 0}$ & 20.97 & 19.68 & 37.11 \\
\hline Cluster 3 & & & $\mathbf{0 . 0 0}$ & 36.60 & 15.54 \\
\hline Cluster 4 & & & & $\mathbf{0 . 0 0}$ & 37.41 \\
\hline Cluster 5 & & & & & $\mathbf{0 . 0 0}$ \\
\hline
\end{tabular}

Bold figures indicate intra-cluster $D^{2}$ value

Table.8 Cluster means for different characters in amaranthus

\begin{tabular}{|c|c|c|c|c|c|c|c|c|c|c|}
\hline Cluster No. & $\begin{array}{c}\text { Days to } \\
50 \% \\
\text { Flowering }\end{array}$ & $\begin{array}{c}\text { Days to } \\
\text { Maturity }\end{array}$ & $\begin{array}{l}\text { Plant } \\
\text { height } \\
\text { (cm) }\end{array}$ & $\begin{array}{l}\text { Inflorescence } \\
\text { Length (cm) }\end{array}$ & $\begin{array}{c}\text { Branches/ } \\
\text { Plant }\end{array}$ & $\begin{array}{c}\text { Biological } \\
\text { Yield/ } \\
\text { Plant }\end{array}$ & $\begin{array}{c}\text { Harvest } \\
\text { Index } \\
(\%)\end{array}$ & $\begin{array}{c}\text { Seed } \\
\text { Volume } \\
\text { Weight } \\
\text { (g/10ml ) }\end{array}$ & $\begin{array}{c}\text { Protein } \\
\text { Content } \\
(\%)\end{array}$ & $\begin{array}{c}\text { Seed } \\
\text { Yield/ } \\
\text { Plant (g) }\end{array}$ \\
\hline Cluster 1 & $77.77 * *$ & 167.30 & $82.29 * *$ & $52.98 * *$ & $4.21 *$ & 117.60 & 8.93 & 6.10 & 12.00 & 9.91 \\
\hline Cluster 2 & 77.10 & $164.53 *$ & 60.69 & 39.01 & 4.57 & $82.37 *$ & $11.77 * *$ & 6.06 & 12.15 & 8.66 \\
\hline Cluster 3 & 75.33 & 166.00 & 76.60 & 48.60 & $6.93 * *$ & 99.00 & 8.55 & $6.00 *$ & $13.20 * *$ & $8.37 *$ \\
\hline Cluster 4 & 75.67 & 164.67 & $49.80 *$ & $31.33^{*}$ & 5.67 & 110.67 & 8.84 & 6.20 & $10.27^{*}$ & 9.63 \\
\hline Cluster 5 & $74.67 *$ & $167.33^{* *}$ & 74.93 & 50.13 & 6.67 & $176.00 * *$ & $5.93 *$ & $6.23 * *$ & 12.17 & $10.27 * *$ \\
\hline
\end{tabular}

*** indicates lower and higher value respectively 
Table.9 Contribution of ten traits of Amaranthus towards divergence

\begin{tabular}{|c|c|c|c|}
\hline S.No. & Source & Times Ranked 1st & Contribution \% \\
\hline $\mathbf{1}$ & Days to 50\% Flowering & 34 & $13.44 \%$ \\
\hline $\mathbf{2}$ & Days to Maturity & 30 & $11.86 \%$ \\
\hline $\mathbf{3}$ & Plant Height $(\mathrm{cm})$ & $\mathbf{8 1}$ & $\mathbf{3 2 . 0 2} \%$ \\
\hline $\mathbf{4}$ & Inflorescence Length $(\mathrm{cm})$ & 6 & $2.37 \%$ \\
\hline $\mathbf{5}$ & Branches/ Plant & 5 & $1.98 \%$ \\
\hline $\mathbf{6}$ & Biological Yield/ Plant & 39 & $15.42 \%$ \\
\hline $\mathbf{7}$ & harvest Index (\%) & 26 & $10.28 \%$ \\
\hline $\mathbf{8}$ & Seed Volume Weight $(\mathrm{g} / 10 \mathrm{ml})$ & 1 & $0.40 \%$ \\
\hline $\mathbf{9}$ & Protein Content $(\%)$ & 31 & $12.25 \%$ \\
\hline $\mathbf{1 0}$ & Seed Yield/ Plant $(\mathrm{g})$ & $\mathbf{0 . 0 1}$ & $\mathbf{0 . 0 0} \%$ \\
\hline
\end{tabular}

Table.10 Most promising amaranthus genotypes

\begin{tabular}{|c|c|c|c|}
\hline $\mathbf{S . N}$ & Characters & $\begin{array}{c}\text { No. of Most } \\
\text { desirable } \\
\text { genotypes }\end{array}$ & Most desirable genotypes \\
\hline $\mathbf{1}$ & Days to 50\% flowering & 6 & RMA 58, SKGPA 86, Ambika GA 12-1, SKGPA 91, SKGPA 72, SKNA \\
\hline $\mathbf{2}$ & Days to maturity & 7 & $\begin{array}{c}\text { 1003 } \\
\text { SKNA 1003, KBGA 5, SKGPA 72, RMA 59, SKGPA 68, RGA 10, } \\
\text { Ambika GA 12-1 }\end{array}$ \\
\hline $\mathbf{3}$ & Plant height $(\mathrm{cm})$ & 3 & RMA 57, BGA 4-9, RGA 72 \\
\hline $\mathbf{4}$ & Inflorescence length $(\mathrm{cm})$ & 5 & RMA 57, RMA 58, RGA 72, BGA 4-9, RGA 11 \\
\hline $\mathbf{5}$ & Number of branches per plant & 7 & SKGPA 91, SKGPA 86, RGA 72, SKGPA 68, SKGPA 72, SKGPA 74, \\
\hline $\mathbf{6}$ & Biological yield per plant $(\mathrm{g})$ & 5 & RGA 11 \\
\hline $\mathbf{7}$ & Harvest index $(\%)$ & 5 & KGPA 86, BGA 8-5, GA 2, BGA 2,RMA 58 \\
\hline $\mathbf{8}$ & Seed volume weight $(\mathrm{g} / 10 \mathrm{ml})$ & 5 & RGA 72, SKNA 403, SKGPA 86, SKGPA 68, GA 2 \\
\hline $\mathbf{9}$ & Protein content $(\%)$ & 3 & RGA 11, SKGPA 74, SKGPA 91 \\
\hline $\mathbf{1 0}$ & Seed yield per plant $(\mathrm{g})$ & 5 & RMA 58, RGA 72, BGA 8-5, Ambika GA 12-1, SKGPA 86 \\
\hline
\end{tabular}


The seed volume weight recorded positive and highly significant correlation with days to maturity (0.3252) and positive correlation with inflorescence length (0.2059), plant height (0.1898), seed yield per plant (0.1656), biological yield /plant (0.1502), number of branches/plant (0.1445) and protein content (0.0173) while negative correlation with days to $50 \%$ flowering $(-0.0942)$, harvest index (0.0336). The protein content recorded negative correlation with biological yield/plant $(-0.2175)$, seed yield per plant (-0.0369) while positive correlation with inflorescence length (0.2231), plant height (0.2200), harvest index (0.1659), number of branches/plant (0.1567) days to maturity (0.0572), seed volume weight (0.0173), days to 50\% flowering (0.0001).

The direct and indirect effects of different characters on seed yield per plant were estimated by path coefficient analysis using simple correlations as given in Table 5. The highest positive direct effect on seed yield per plant was exhibited by biological yield per plant (1.1094) followed by harvest index (1.0279) and negative direct effect on seed yield / plant was exhibited by days to $50 \%$ flowering (-0.1037) followed by number of branches per plant $(-0.0714)$, days to maturity $(-0.0258)$, protein content $(-0.0168)$ and seed volume weight $(-0.0145)$. These characters have also identified as major direct contributors of yield by Rymuza et al., (2012), Mollasadeghi et al., (2011). The highest negative indirect effect on seed yield /plant was exhibited by biological yield / plant via harvest index (-0.8984) followed by harvest index via days to $50 \%$ flowering $(-0.5409)$, inflorescence length via harvest index (0.0597) and plant height via harvest index (0.0293). The highest positive indirect effect on seed yield / plant was exhibited by biological yield per plant via days to maturity (0.7148) followed by harvest index via protein content (0.1706), inflorescence length via plant height (0.1634), and plant height via inflorescence length (0.0852). Some of the earlier reports have also identified these characters as important indirect contributor towards the expression of seed yield in amaranthus (Ayehu et al., 2015; Keshav et al., 2013; Haghighi et al., 2012). The remaining estimates of the indirect effects in the present analysis were too low to be considered important. The residual effect observed was 0.6134 which indicates that some of the characters which might contribute to yield have not been included in the study.

Then Mahalanobis $\mathrm{D}^{2}$ analysis was employed to study genetic diversity existing among 23 amaranthus genotypes on the basis of ten quantitative characters. The pseudo F-test revealed that five clusters arrangement was the most appropriate for grouping the 23 genotypes. Therefore, the 23 genotypes were grouped into five different non overlapping clusters. The distribution of 23 amaranthus genotypes in five clusters is presented in Table 6 . The highest number of genotypes appeared in cluster 5 which contained 7 genotypes followed by cluster $3 \&$ cluster 4 having 5 genotypes. Clusters 2 having 4 genotypes and cluster 1 having 2 genotypes.

The estimates of average intra and inter cluster distance for the five cluster is presented in Table 7. The highest intra-cluster distance was recorded in cluster 2 (12.20) followed by cluster 1 (11.58), while the lowest value was recorded in case of cluster 3,4 and 5 (0.00). The maximum inter cluster distance was recorded between cluster 1and cluster 4 (39.44) followed by cluster 4 and cluster 5 (37.41). The minimum inter cluster distance was observed between cluster 3 and cluster 5 (15.54) followed by cluster 2 and cluster 4 (19.68).

The mean performance of clusters, for ten characters is presented in Table 8 . The cluster 1 showed high mean performance for plant 
height (82.29), days to $50 \%$ flowering (77.77) and inflorescence length (52.98) while other exhibited average mean performance for remaining characters. The cluster 2 showed high mean performance for harvest index (11.77) and low mean performance for days to maturity (164.53), biological yield per plant (82.37) while other character showed average mean performance. The cluster 3 showed high mean performance for protein content (13.20), number of branches per plant (6.93) and showed low mean performance for seed yield per plant (8.37) and seed volume weight (6.00). The other characters showed average mean performance in cluster 3 . The cluster 4 showed no high mean performance while it showed low mean performance for plant height (49.80), inflorescence length (31.33), protein content (10.27) and average performance for other characters. The cluster 5 showed high mean performances for biological yield per plant (176.00), days to maturity (167.33), seed yield per plant (10.27) while it showed low mean performance for days to $50 \%$ flowering (74.67), harvest index (6.23) and other characters showed average performance in cluster 5 .

The contribution of ten characters towards divergence (Table 9) showed the highest contribution by plant height (32.02\%) followed by biological yield per plant $(15.42 \%)$. While the seed yield per plant $(0.00 \%)$ showed lowest contribution towards divergence.

The Most promising amaranthus genotypes for ten characters are given in Table-10. The highest seed yield per plant was obtained in RMA 58, RGA 72, BGA 8-5, Ambika GA 121 and SKGPA 86. Early days to $50 \%$ flowering reveled by RMA 58, SKGPA 86, Ambika GA 12-1, SKGPA 91, SKGPA 72 and SKNA 1003. Early maturity showed by SKNA 1003, KBGA 5, SKGPA 72, RMA 59, SKGPA 68, RGA 10 and Ambika GA 12-1.
The highest plant height was obtained in RMA 57, BGA 4-9 and RGA 72. The highest inflorescence length was exhibited by RMA 57, RMA 58, RGA 72, BGA 4-9 and RGA 11. The highest number of branches per plant was reported in SKGPA 91, SKGPA 86, RGA 72, SKGPA 68, SKGPA 72, SKGPA 74 and RGA 11. The highest biological yield per plant obtained in SKGPA 86, BGA 8-5, GA 2, BGA 2 and RMA 58. The highest harvest index was obtained in KBGA 5, SKNA 1003, RGA 10, RMA 59 and SKNA 403. The maximum seed volume weight was found in RGA 72, SKNA 403, SKGPA 86, SKGPA 68 and GA 2. The highest protein content was reported in RGA 11, SKGPA 74 and SKGPA 91. These are elite/most promising evaluated and identified genotype of amaranthus. Identified superior/elite/most promising genotypes having disease resistance and wider adaptability with maximum production coupled high protein content can be further utilized in breeding programme for improvement of amaranthus genotypes.

From all over the analysis, it can be concluded that high amount of variability was present in the genotypes under study which provided a better opportunity to select desirable genotypes for further utilization in breeding programme. Correlation and path analysis indicated that inflorescence length, plant height, biological yield per plant and harvest index had true relationship with seed yield and they are the major yield contributing traits. The characters identified above was important yield contributing components merit due consideration in formulating selection strategy in amaranthus for selecting high yielding genotypes. Hence, direct selection for these traits would be rewarding for yield improvement in amaranthus. Therefore, RMA 58, RGA 72, BGA 8-5, Ambika GA 12-1 and SKGPA 86 exhibiting higher mean performance for seed yield per plant, are the elite genotypes. These genotypes can be 
further utilized in breeding programme for crop improvement regarding seed yield per plant in amaranthus.

\section{References}

Akaneme FI and Ani GO. 2013. Morphological Assessment of Genetic Variability among Accessions Amaranthus hybrids. World Applied Sciences Journal 28 (4): 568-577.

Araus JI, Bor, J, CecCadelli S and Grando S. 1997. Relationship between leaf structure and carbon isotope discrimination in field grown barley. Plant Physiol. Biochem. 35: 533-541.

Ayehu FH, Lal S and Sinatayehu A. 2015. Estimation of Association Characters in Amaranths Germplasm Accessions (Amaranthus Spp.) under Mizan and Tepi Condtions, South West Ethiopia, 2 (1) 2348-6848.

Becker R, Wheeler EL, Lorenz K, Stafford AE, Grosjean OK, Betschart AA and Saunders RM. 1981. A composition study of amaranth grain. J Food Sci, 46:1175-1180.

Breene WM. 1991. Food uses of grain amaranth. Cereal Food World 36: 426-430.

Bressani R. 1989. The proteins of grain amaranth. Food Rev. Int., 5:13-38.

Burton GW and de Vane EH. 1953. Estimating heritability in tall fescue (Festuca arundinacea) from replicated clonal material Agron.J.45:478-481.

Haghighi ML, Abbaszadeh B, Lebaschi MH and Shahrebabaki MAV. 2012. Evaluating effective traits on yield of two medicinal amaranths (Amaranthus hypochondriacus L. var. Cim and var. Kharkofski) in Karaj, Iran. Annals of Biological Research, 3 (2):1014-1019.

Johnson HW, Robinson HF and Comstock RE. 1955. Estimates of genetic and environmental variability in soybean.
Agron. J., 47: 477-483.

Kadoshnikov SI, Kadoshnikova IG, Martirosyan DM. 2005. Investigation of Fractional Composition of the Protein in Amaranth, Non-Traditional Natural Resources, Innovation Technologies and Products, Russian Academy of Natural Sciences, Moscow 12: 81-104.

Keshav KU and Maurya K. 2013. Genetic association between foliage yield and its biochemical parameters in vegetable Amaranthus (Amaranthus tricolor) International Journal of Advanced Research and Technology. 1: 56-74.

Kumar R, Bhushan B, Pal R and Gaurav SS. 2014. Correlation and path coefficient analysis for quantitative traits in wheat (Triticum aestivum L.) under normal condition. Annuals of Agri Bio Research, 19(3):447-450.

Lehman JW. 1989. Proteins of grain amaranth, Amer. Amaranth Inst., Legacy 2:3-6.

Lehman JW. 1996. Case history of grain amaranth as an alternative crop. Cereal Food World 41: 399-411.

Mahalanobis PC. 1936. A statistical study at Chinese head measurements. J. Asiatic Soc. Bengal. 25: 301-377.

Martirosyan DM. 2001. Amaranth as a Nutritional Supplement for the Modern Diet. Amaranth Legacy, USA, 14: 2-4.

Martirosyan DM. 2003. Amaranth, Quinoa and Lentils as a Source of Modern Diet and Functional Foods, NonTraditional Natural Resources, Innovation Technologies and Products Moscow, Russia, Russian Academy of Natural Sciences, 91-100.

Mollasadeghi V, Imani AA, Shahryari R and Khayatnezhad M. 2011. Correlation and path analysis of morphological traits in different wheat genotypes under end drought stress condition. Middle East J. Scientific Res., 7 (2): 
221-224.

Pan R and Dong Y. 1995. Photosynthesis and respiration-In: Pan R, Y Dong, Plant Physiology 3rd Ed. Higher educational press, Beijing, 67-143.

Rymuza K, Turska E, Wielogorska G, Wyrzykowska $\mathrm{M}$ and Bombik A. 2012. Evaluation of yield determination of spring wheat grown in monoculture interrupted with stubble crop growth by means of path analysis. Acta Scientiarum Polonorum - Agricultura., 11 (2): 53-61.

Sauer JD. 1993. Amaranthaceaes Amaranth family. In Historical Geography of Crop Plants: a Select Roster; CRC Press: Boca Raton, FL; 9-14.

Singh BN, Soni SK, Srivastava A and Yadav VK. 2012. Multivariate analysis to architecture model plant type for saline soil in bread wheat (Triticum aestivum L. em Thell.). Environment and Ecology, 30 (1): 179-182.

Teutonico RA and Knorr D. 1985. Amaranth: composition, properties, and applications of a rediscovered food crop. Food Technol, 39: 49-60.

Thomas JA, Jeffrey AC, Atsuko K and David MK. 2005. Regulating the proton budget of higher plant photosynthesis. Proc. Natl. Acad. Sci. USA 102: 97099713.

Williams JT, Brenner D. 1995. Grain amaranth (Amaranthus species), Cereals and pseudocereals. Ed by Williams JT., Chapman and Hall. London. 129-186.

\section{How to cite this article:}

Shiv Prakash Shrivastav, C. B. Yadav, Vartika Singh and Virendra Maurya. 2020. Evaluation and Identification of most Promising Genotypes for Varietal Development in Amaranthus (Amaranthus paniculatus L.). Int.J.Curr.Microbiol.App.Sci. 9(01): 345-360. doi: https://doi.org/10.20546/ijcmas.2020.901.039 